\title{
EVALUACIÓN DEL DESEMPEÑO DEL MODELO WRF SOBRE LA REGIÓN MONTAÑOSA CENTRAL DE VERACRUZ, MÉXICO, DURANTE UNA CAMPAÑA DE MEDICIONES EN EL VERANO DEL 2015
}

\author{
Juan Matías MÉNDEZ PÉREZ1 ${ }^{1}$ Ana Karen RAMÍREZ LÓPEZ1, \\ y Ana Ceres LUÉVANO DE LA CRUZ ${ }^{1}$ \\ ${ }^{1}$ Grupo de Climatología Aplicada de la Universidad Veracruzana. México. \\ jumendez@uv.mx , a.karenr190@gmail.com, anaceres25@gmail.com
}

\section{RESUMEN}

La región montañosa central de Veracruz, México, se caracteriza por estar influenciada por su orografía y cercanía al mar (Golfo de México), lo que permite estudiar el acoplamiento entre las diferentes capas atmosféricas y los sistemas de circulación que la afectan durante el año. Mediante mediciones en superficie y en altura, se pretende conocer mejor el transporte de humedad de la costa hacia el altiplano, además de validar las parametrizaciones de los flujos verticales de momento, energía y masa en un modelo numérico de mesoescala. Para ello, de manera simultánea a la campaña de mediciones (27 de junio - 4 de julio de 2015) en la región, se realizó un conjunto de 14 simulaciones numéricas a alta resolución espacial $(1 \mathrm{~km})$ y temporal $(1 \mathrm{~h})$ con diferentes opciones de parametrizaciones físicas del modelo Weather Research and Forecasting (WRF). Esto con la finalidad de evaluar el desempeño del WRF para reproducir las condiciones atmosféricas locales registradas durante dicha campaña. El mejor desempeño del modelo se obtuvo con las configuraciones de capa límite planetaria.

Palabras clave: Veracruz, modelo WRF, capa límite planetaria, interacción superficie-atmósfera.

\begin{abstract}
The central mountainous region of Veracruz, Mexico, is characterized by being influenced by its topography and proximity to the sea (Gulf of Mexico), allowing to study the link between the different atmospheric layers and circulation systems that affect it during the year. By surface and upper aire measurements, it is to better understand the transport of moisture from the coast to the highlands, and to validate the settings of the vertical flows of momentum, energy and mass in a mesoscale numerical model. To do this, simultaneously with a measurement campaign (June 27 - July 4, 2015) way in the region, a set of 14 numerical simulations were performed at high spatial $(1 \mathrm{~km})$ and temporal $(1 \mathrm{~h})$ resolution with different physical parameterizations options of Weather Research and Forecasting (WRF) model. This in order to evaluate the performance of WRF to reproduce the local weather conditions during the campaign. The best performance was obtained with model configurations planetary boundary layer.
\end{abstract}


Key words: Veracruz, WRF model, planetary boundary layer, land surfaceatmosphere interaction.

\section{INTRODUCCIÓN}

La zona montañosa central del Golfo de México se caracteriza por una combinación de cadenas montañosas que forman parte del Eje Neovolcánico, la Sierra Madre Oriental y la Sierra Madre del Sur, así como de planicies, llanuras, lomeríos y valles. Las elevaciones más altas corresponden al Pico de Orizaba o Citlaltépetl con 5,700 msnm y el volcán del Cofre de Perote, con una altura de 4,280 msnm. Este importante gradiente altitudinal, es uno de los más escarpados del mundo, pues va de los 5,700 m de altitud (cima del Pico de Orizaba) al nivel del mar en una distancia horizontal de 100 km (Fitzjarrald, 1984 y 1986).

Como consecuencia, la circulación sinóptica se ve influenciada por la orografía; por ejemplo en el semestre centrado en el verano los vientos alisios encuentran la pared montañosa, ascienden de manera forzada para producir precipitación de más de $1,000 \mathrm{~mm}$ a barlovento y menos de $400 \mathrm{~mm}$ a sotavento. Durante el invierno, los vientos intensos y frecuentes en la región son provocados por los llamados nortes -invasiones de aire polar con descensos de temperatura de entre 2 y $5^{\circ} \mathrm{C}$ en la costa-, mientras que en los meses de verano y otoño los ciclones tropicales son un factor presente en Veracruz, provocando una intensificación en la precipitación como resultado de su orografía (Jáuregui y Zitácuaro, 1995).

Los sistemas de circulación que afectan a la zona montañosa central del Golfo de México en distintas épocas del año, la compleja orografía que presenta, así como su cercanía al mar, convierten a esta región en un laboratorio natural para explorar el acoplamiento entre las diferentes capas atmosférica (superficial y de mezcla), la capa nubosa y la circulación sinóptica, mediante el uso de información existente y de simulaciones numéricas a través de la implementación de modelos de mesoescala.

Dentro del área de estudio destaca la zona de Laguna Verde, es el punto donde se enfoca la evaluación del modelo WRF del presente trabajo. La Central Nucleoeléctrica Laguna Verde (LV) se ubica en la costa central del Golfo de México, en el punto donde el eje Neovolcánico y la Sierra Madre Oriental llegan hasta la costa.

Para poder comprender la manera en que se relacionan las características de la capa atmosférica superficial y el comportamiento de la capa de mezcla se requiere de un conjunto de datos medidos y datos con aproximaciones numéricas en la región de estudio; sobre todo cuando dichas capas se encuentran bajo la influencia de diversos sistemas de circulación regional o sinóptica dominantes (vientos alisios, alisios perturbados, nortes o el anticiclón de las Bermudas-Azores).

El objetivo es evaluar el desempeño del modelo Weather Research and Forecasting (WRF) para simular, a alta resolución espacial (hasta $1 \mathrm{~km}$ ) y temporal $(1 \mathrm{~h})$, las condiciones atmosféricas en la vertiente del Golfo de México y su interacción con la topografía compleja de la zona durante el periodo de mediciones ( 27 de junio -4 de julio del 2015). Así como determinar la configuración del modelo y las parametrizaciones físicas adecuadas para el análisis de la interacción superficie/atmósfera. Con ello, se pretende evaluar sistemáticamente el desempeño y las limitaciones del mode- 
lo WRF para simular los sistemas meteorológicos de mesoescala y su interacción con la topografía compleja en el área de estudio.

El enfoque de este estudio es la comparación de las condiciones atmosféricas en los niveles cercanos a la superficie del suelo y su interacción con la topografía. Debido a esto, el parámetro físico del modelo WRF de mayor interés será el relacionado a la capa límite planetaria. Dicha parametrización cuenta con diversos esquemas que resuelven particularmente la transferencia de energía turbulenta y de humedad de la superficie.

\section{MÉTODOS}

\subsection{Descripción del modelo WRF}

El modelo llamado Weather Research and Forecasting (WRF) (Skamarock et al. 2008) es un modelo numérico de área limitada de última generación diseñado para la investigación y la predicción numérica del tiempo meteorológico. Este modelo permite simulaciones tanto de condiciones atmosféricas reales (a través de análisis y observaciones) como idealizadas, a diferentes escalas. Dada su flexibilidad y constantes mejoras, el WRF actualmente es uno de los modelos de mayor uso en diversos centros meteorológicos y de investigación alrededor del mundo.

\subsection{Descripción de los datos}

En este estudio, las condiciones iniciales y de frontera del modelo WRF fueron suministrados a través de los datos del llamado Análisis Final de NCEP del Sistema de Pronóstico Global (GFS, por sus siglas en inglés) con una resolución espacial de $1^{\circ} \mathrm{x} 1^{\circ} \mathrm{y}$ temporal de 6 horas.

Para el proceso de comparación y evaluación del desempeño del modelo WRF, se utilizaron datos horarios de temperatura a $2 \mathrm{~m}$, viento (dirección y magnitud) a 10 m, y humedad relativa a 2 m, registrados en Laguna Verde $\left(19^{\circ} 43.6^{\prime} \mathrm{N}, 96^{\circ} 24^{\prime} \mathrm{W}, 14\right.$ $\mathrm{msnm})$. Dicha estación formó parte de una red de 18 sitios durante la campaña de mediciones realizada entre el 27 de junio y el 4 de julio del 2015 en la región de estudio.

\subsection{Diseño de las simulaciones numéricas}

Las simulaciones numéricas se realizaron con el modelo WRF en su versión 3.5.1 para un dominio madre, D1, con una resolución espacial de $20 \mathrm{~km}$, y dos dominios anidados, D2 y D3, con resolución espacial de 5 y 1 km, respectivamente (Fig. 1). El dominio madre está centrado en $19.70^{\circ} \mathrm{N}$ y $96.00^{\circ} \mathrm{W}$, con una proyección de mapa tipo Mercator, y 27 niveles eta en la vertical.

El modelo WRF cuenta con diferentes opciones para la parte física, este trabajo se concentra en los esquemas de parametrizaciones de cúmulus $(\mathrm{cu})$, microfísica $(m p)$, capa límite planetaria $\left(b l \_b l\right)$, capa superficial ( $\left.s f \_f c l a y\right)$, superficie terrestre (sf_surface) y arrastre en superficie (topo_wind). Las opciones físicas consideradas en este trabajo se enlistan en la Tabla 1. 


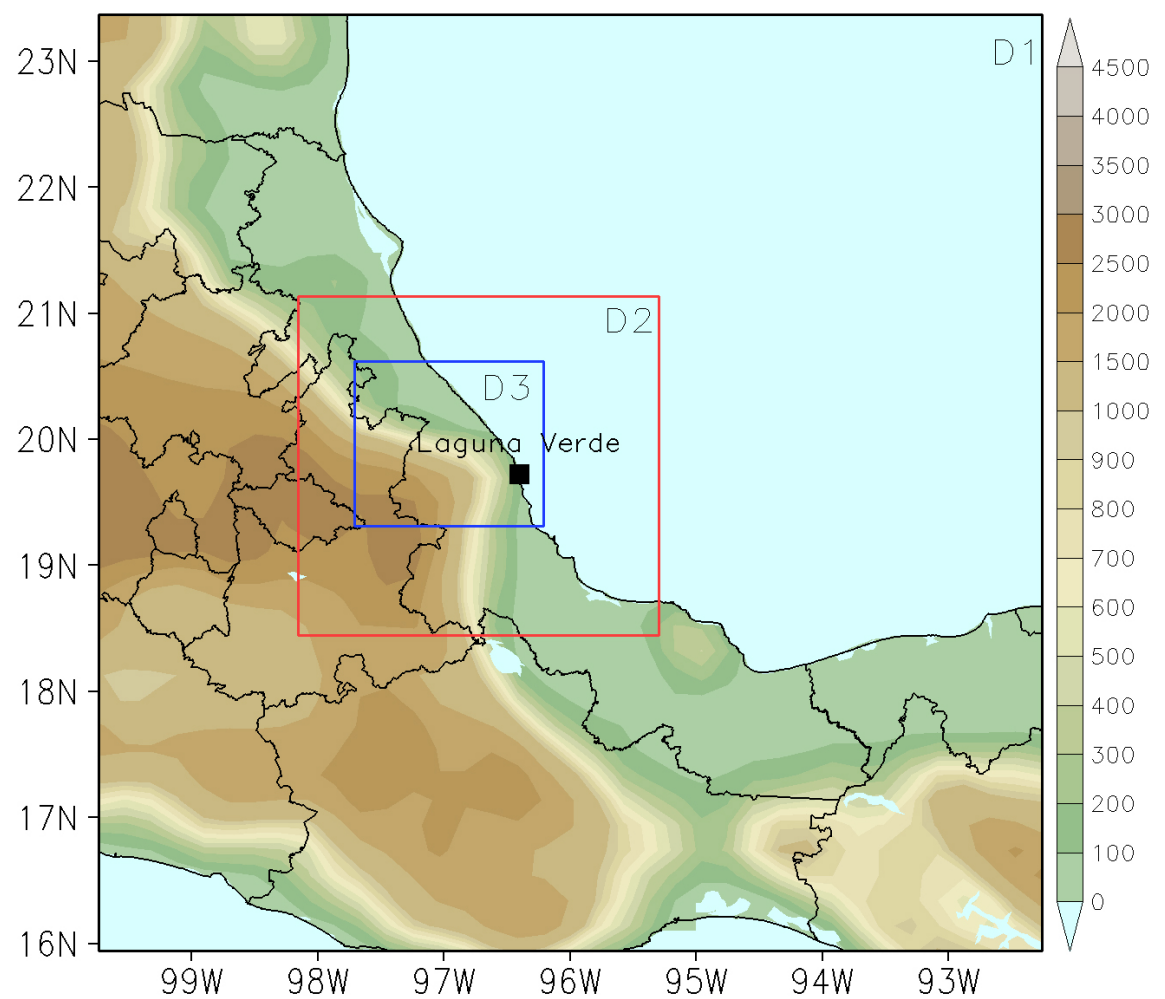

Fig. 1: Dominio madre D1 (20 km, $38 x 40)$ y anidados D2 (5 km, $57 x 57)$ y D3 (1 km, $146 x 136)$ del área de estudio y topografía en m del modelo. Entre paréntesis se indica la resolución y el número de puntos de malla.

Se realizaron tres simulaciones con cambios en la parte cúmulus, tres más con modificaciones en el parámetro de microfísica, la parametrización de mayor interés fue la capa límite planetaria por lo cual se elaboraron cuatro experimentos; las opciones físicas requerían forzosamente un cambio en el parámetro de capa superficial; otro experimento se hizo para superficie terrestre y tres más para el parámetro de arrastre en superficie. Las parametrizaciones de radiación de onda cartay onda larga se utilizaron los esquemas de Dudhia y el de Transferencia Radiativa Rápida (RRTM), respectivamente, fueron siempre las mismas para todos los experimentos.

Se realizó una sola integración numérica en el tiempo desde el día 27 de junio a las $00 \mathrm{Z}$ ( 26 de junio a las $7 \mathrm{pm}$ hora local) hasta el día 6 de julio a las $00 \mathrm{Z}$ ( 5 de julio a las 7 pm) del 2015, para cada una de las 14 configuraciones (Tabla 2). Se utilizó la opción de reinicio cada 24 horas hasta cubrir el periodo deseado. Las primeras 24 horas de integración se eliminaron para evitar el problema de estabilización del modelo. Las salidas numéricas del dominio de interés (D3) corresponden a valores medios en lapsos de 60 minutos. 


\begin{tabular}{|c|c|}
\hline Parametrización & Esquema \\
\hline Cúmulus $(c u)$ & $\begin{array}{l}\text { Sin Cúmulus }(S C) \\
\text { Kain-Fritsch (new Eta) }(K F) \\
\text { Betts-Miller-Janjic }(B M J)\end{array}$ \\
\hline Microfísica $(m p)$ & $\begin{array}{l}\text { Lin et al. }(\text { LIN) } \\
\text { WSM de } 3 \text { clases (WSM3) } \\
\text { WSM de } 6 \text { clases (WSM6) }\end{array}$ \\
\hline Capa límite planetaria $\left(b l \_p l\right)$ & $\begin{array}{l}\text { Yonsei University }(Y S U) \\
\text { TKE Mellor-Yamada-Janjic Eta (MYJ) } \\
\text { NCEP-GFS }(G F S) \\
\text { ACM2 Pleim }(A C M 2)\end{array}$ \\
\hline Capa superficial (sf_sfclay) & $\begin{array}{l}\text { Semejanza Monin-Obukhov-MM5 (MOM) } \\
\text { Semejanza Monin-Obukhov-Janjic }(M O J) \\
\text { NCEP-GFS }(G F S)\end{array}$ \\
\hline Superficie terrestre (sf_surface) & $\begin{array}{l}\text { Difusión termal MM5 (MM5) } \\
\text { NCEP-Noah }(N O A H)\end{array}$ \\
\hline Arrastre en superficie (topo_wind) & $\begin{array}{l}\text { Sin arrastre en superficie }(S A) \\
\text { Jiménez-Dudhia }(J D) \\
\text { Mass-Ovens }(M O)\end{array}$ \\
\hline
\end{tabular}

Tabla 1: Parametrizaciones físicas y esquemas analizados del modelo WRF. Entre paréntesis se indica la nomenclatura usada en la Tabla 2.

\begin{tabular}{|c|c|c|c|c|c|c|}
\hline Experimento & cu & mp & bl_pbl & sf_sfclay & sf_surface & topo_wind \\
\hline E-01 & $\mathrm{KF}$ & WSM3 & YSU & MOM & MM5 & JD \\
\hline E-02 & BMJ & WSM3 & YSU & MOM & MM5 & JD \\
\hline E-03 & $\begin{array}{c}\mathrm{KF} / \\
\mathrm{BMJ}\end{array}$ & WSM3 & YSU & MOM & MM5 & JD \\
\hline E-04 & $\mathrm{KF}$ & $\begin{array}{l}\text { WSM3/ } \\
\text { WSM6 }\end{array}$ & YSU & MOM & MM5 & JD \\
\hline E-05 & $\mathrm{KF}$ & LIN & YSU & MOM & MM5 & JD \\
\hline E-06 & $\mathrm{KF}$ & WSM6 & YSU & MOM & MM5 & JD \\
\hline E-07 & $\mathrm{KF}$ & WSM3 & MYJ & MOJ & MM5 & SA \\
\hline E-08 & $\mathrm{KF}$ & WSM3 & ACM2 & MOM & MM5 & SA \\
\hline E-09 & $\mathrm{KF}$ & LIN & MYJ & MOJ & MM5 & SA \\
\hline E-10 & $\mathrm{KF}$ & WSM3 & GFS & GFS & MM5 & SA \\
\hline E-11 & $\mathrm{KF}$ & WSM3 & YSU & MOM & NOAH & JD \\
\hline E-12 & $\mathrm{KF}$ & WSM3 & YSU & MOM & MM5 & SA \\
\hline E-13 & $\begin{array}{l}\mathrm{KF} / \\
\mathrm{SC}\end{array}$ & WSM3 & YSU & MOM & MM5 & JD \\
\hline E-14 & $\mathrm{KF}$ & WSM3 & YSU & MOM & MM5 & $\mathrm{MO}$ \\
\hline
\end{tabular}

Tabla 2: Experimentos realizados y sus parametrizaciones físicas. 
Para el caso particular de la parametrización física de capa límite planetaria se utilizaron tres esquemas, que corresponden a los experimentos E-07 (MYJ), E-08 (ACM2) y E-09 (GFS). Cada uno de estos experimentos se tuvieron que realizar algunas configuraciones adecuadas para el buen funcionamiento (Tabla 2).

\subsection{Evaluación de las simulaciones numéricas}

Existen diversas metodologías para evaluar un modelo, en este caso se consideraron las recomendadas en trabajos análogos al presente (Gómez-Navarro et al. 2015, Singh et al. 2015, Reboredo et al. 2015). Para obtener un valor global del periodo completo, se calcularon los errores estadísticos denominados: error absoluto medio (MAE), el sesgo o error medio (BIAS), la raíz cuadrada del error cuadrático medio (RMSE), el porcentaje de error (\% ERROR) y el índice de concordancia (IC) entre los datos horarios observados y estimados de las variables de temperatura, magnitud del viento y humedad relativa; mientras que para la dirección del viento se calculó el error denominado BIASD.

\section{RESULTADOS}

Los resultados de la comparación de las tres configuraciones de la parametrización de la capa límite planetaria son presentados a continuación utilizando los estadísticos propuestos.

Los tres esquemas produjeron resultados similares para la temperatura (Tabla 3), siendo el experimento E-08, cuya configuración utiliza el esquema ACM2 (Modelo Convectivo Asimétrico), el que tuvo un desempeño ligeramente mejor para reproducir la evolución horaria de la temperatura en la región de Laguna Verde. En cuanto a humedad relativa, el experimento E-08 tuvo un mejor desempeño comparado con los otros dos esquemas (E-07 y E-09) los cuales tienen resultados similares. Finalmente, para el caso de la magnitud y dirección del viento, nuevamente el experimento E-08 tuvo un mejor desempeño para esta variable, particularmente para la dirección del viento, cuyo error es significativamente menor.

En la figura 2, se aprecia la evolución horaria (observada y simulada) promedio durante el periodo de la campaña de mediciones en la estación de Laguna Verde. En lo que se refiere a la temperatura a $2 \mathrm{~m}$, los tres experimentos tienen resultados similares. El modelo tiende a sobreestimar, en promedio $1{ }^{\circ} \mathrm{C}$, desde la medianoche hasta las primeras horas de la mañana; a partir de las 9 a.m. el modelo subestima la temperatura en alrededor de $1{ }^{\circ} \mathrm{C}$, siendo menor la diferencia hacia la medianoche. El esquema de ACM2 tiene un desempeño ligeramente mejor a los otros dos esquemas. Por otro lado, la humedad relativa es subestimada, en promedio $5 \%$, desde la media noche hasta las primera horas del día; después de las 9 a.m. el modelo tiende a sobreestimar la humedad relativa, disminuyendo la diferencia entre lo observado y lo simulado hacia la medianoche. De los tres experimentos, el que mejor desempeño tuvo es el que corresponde al esquema de ACM2. Finalmente, para el caso de la magnitud del viento, en los experimentos E-07 y E-09, el modelo subestimó la evolución horaria de la magnitud del viento. Mientras que el experimento E-08 tuvo un mejor desempeño, sobre todo entre las 6 a.m. y 6 p.m. 
Por lo anterior, de acuerdo a esta evaluación a partir de estos estadísticos de error, el esquema ACM2 es considerado el que mejor desempeño tuvo para simular las variables de interés, como se mostró en la Tabla 3 y en la figura 2.

\begin{tabular}{|c|c|c|c|c|}
\hline \multirow{2}{*}{$\begin{array}{c}\text { Parámetros } \\
\text { meteorológicos }\end{array}$} & \multirow{2}{*}{$\begin{array}{c}\text { Errores } \\
\text { estadísticos }\end{array}$} & \multicolumn{3}{|c|}{ Experimentos } \\
\hline & & $E-07$ & $E-08$ & $E-09$ \\
\hline \multicolumn{5}{|l|}{ Temperatura } \\
\hline & $\operatorname{MAE}\left({ }^{\circ} \mathrm{C}\right)$ & 1.401 & 1.367 & 1.448 \\
\hline & $\operatorname{RMSE}\left({ }^{\circ} \mathrm{C}\right)$ & 1.689 & 1.655 & 1.732 \\
\hline & $\operatorname{BIAS}\left({ }^{\circ} \mathrm{C}\right)$ & -0.058 & 0.190 & 0.054 \\
\hline & $\% E R R O R$ & 1.249 & 0.075 & 0.757 \\
\hline & $I C$ & 0.429 & 0.452 & 0.400 \\
\hline \multirow[t]{6}{*}{ Humedad Relativa } & & & & \\
\hline & MAE (\%) & 8.442 & 5.880 & 8.173 \\
\hline & RMSE (\%) & 9.797 & 6.984 & 9.581 \\
\hline & $B I A S(\%)$ & 3.103 & 1.277 & 2.933 \\
\hline & $\%$ ERROR & 4.036 & 0.265 & 3.829 \\
\hline & IC & 0.280 & 0.158 & 0.180 \\
\hline \multirow[t]{6}{*}{ Magnitud del viento } & & & & \\
\hline & $\operatorname{MAE}(\mathrm{m} / \mathrm{s})$ & 1.408 & 1.392 & 1.634 \\
\hline & $R M S E(\mathrm{~m} / \mathrm{s})$ & 1.913 & 1.755 & 2.116 \\
\hline & $B I A S(\mathrm{~m} / \mathrm{s})$ & -1.070 & -0.515 & -1.326 \\
\hline & $\%$ ERROR & 2.215 & 1.857 & 2.779 \\
\hline & $I C$ & 0.515 & 0.557 & 0.263 \\
\hline Dirección del viento & $B I A S D\left({ }^{\circ}\right)$ & 3.135 & 1.399 & 3.648 \\
\hline
\end{tabular}

Tabla 3: Índices estadísticos de error para los tres esquemas de parametrización de capa límite planetaria (ver Tabla 2) en la zona de Laguna Verde. El mejor desempeño para cada estadístico está marcado en gris.

\section{DISCUSIÓN}

Un total de catorce experimentos de sensibilidad fueron realizados sobre la zona montañosa central del Golfo de México, variando los diferentes esquemas de parametrizaciones físicas de cúmulus, capa límite planetaria, microfísica, capa superficial, superficie terrestre y arrastre en superficie, durante la campaña de mediciones realizada en la zona entre el 27 de junio y el 5 de julio del 2015. Esto con la finalidad de encontrar la configuración óptima del modelo WRF para reproducir las variaciones horarias de temperatura, humedad relativa y viento (magnitud y dirección) en la zona de estudio. Este trabajo se ha enfocado en los esquemas de capa límite planetaria debido a su relevancia para simular los intercambios de flujos entre la superficie y la atmósfera. 
Se ha evaluado las diferencias de variables meteorológicas de interés (temperatura, humedad relativa, magnitud y dirección del viento) comparado con observaciones siguiendo un análisis estadístico. Los resultados obtenidos de este análisis estadístico, para los tres esquemas de parametrizaciones de capa límite planetaria, muestran que no hay diferencias significativas para la temperatura, mientras que los mejores resultados se obtuvieron para la humedad relativa y la magnitud del viento, destacando por su desempeño el esquema ACM2 (Modelo Convectivo Asimétrico).
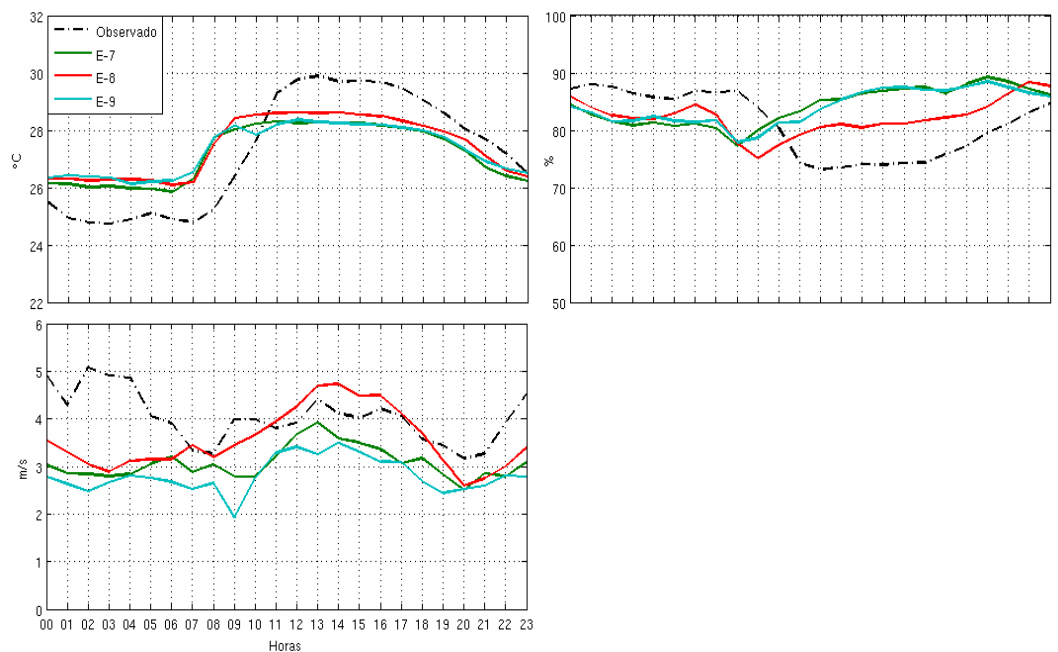

Fig. 2: Evolución horaria promedio de temperatura a $2 \mathrm{~m}\left(\mathrm{en}{ }^{\circ} \mathrm{C}\right)$, humedad relativa a $2 \mathrm{~m}$ (en \%), y magnitud del viento (en $\mathrm{m} / \mathrm{s}$ ) a $10 \mathrm{~m}$ observada y simulada para los tres esquemas de parametrización de capa límite planetaria (ver Tabla 2) en Laguna Verde.

\section{AGRADECIMIENTOS}

Este trabajo ha sido desarrollado y financiado por el proyecto de investigación "Interacción superficie/atmósfera en la zona montañosa central de la vertiente del Golfo de México: observaciones y modelación a alta resolución” de Ciencia Básica de SEP-CONACYT CB-2012-01;000000000183040.

\section{REFERENCIAS}

Fitzjarrald, D. (1984). Katabatic wind in opposing flow. Journal of the Atmospheric Sciences, 41 (7), 1143-1158. doi: http://dx.doi.org/10.1175/15200469(1984)041<1143:KWIOF>2.0.CO;2

Fitzjarrald, D. (1986). Slope winds in Veracruz. Journal of Climate and Applied Meteorology (25), 133-144. doi: http://dx.doi.org/10.1175/15200450(1986)025<0133:SWIV >2.0.CO;2

Gómez-Navarro, J.J., Raible, C.C., \& Dierer, S. (2015). Sensitivity of the WRF model to PBL parameterisations and nesting techniques: evaluation of wind storms over complex terrain. Geoscientific Model Development, 8(10), 3349-3363. doi: $\underline{\text { http://dx.doi.org/10.5194/gmdd-8-5437-2015 }}$ 
Jáuregui, E. e I. Zitácuaro (1995). El impacto de los ciclones tropicales del Golfo de México, en el estado de Veracruz. La Ciencia y el Hombre, No. 21: 75-119. Recuperado de http://cdigital.uv.mx/bitstream/123456789/5331/2/199521P75. pdf

Reboredo, B., Arasa, R., \& Codina, B. (2015). Evaluating Sensitivity to Different Options and Parameterizations of a Coupled Air Quality Modelling System over Bogotá, Colombia. Part I: WRF Model Configuration. Open Journal of Air Pollution, 4(2), 47-64. doi: http://dx.doi.org/10.4236/ojap.2015.42006

Ruiz-Barradas, A., Tejeda-Martínez, A., Miranda-Alonso, S. \& Flores-Zamudio R.H. (2010). Climatología en Atlas del patrimonio natural, histórico y cultural de Veracruz. Gobierno del estado de Veracruz. México. Recuperado de http:// cdigital.uv.mx/bitstream/123456789/9649/1/03CLIMATOLOGIA.pdf

Singh, J., Yeo, K., Liu, X., Hosseini, R., \& Kalagnanam, J.R. (2015). Evaluation of WRF model seasonal forecasts for tropical region of Singapore. Advances in Science and Research, 12, 69-72. doi: http://dx.doi.org/ 10.5194/asr-12-69-2015

Skamarock, W.C., Klemp, J.B., Dudhia, D.O., Gill, D.M., Barker, W., Wang, W. \& Powers, J.G. (2008). A description of the advanced research WRF Version 3. doi: http://dx.doi.org/10.5065/D68S4MVH 